\title{
Statistical learning applied to computer-assisted fish age and growth estimation from otolith images
}

\author{
Ronan Fablet
}

Ifremer/LASAA, BP 70, 29280 Plouzané, France ; rfablet@ifremer.fr

\begin{abstract}
Computer-assisted tools need to be developed to help in the accurate and efficient acquisition of fish age and growth data for ecological and assessment issues. Stating fish age and growth analysis as pattern classification issues, the proposed approach relies on a statistical learning strategy. Given otolith images interpreted by an expert, probabilistic kernel-based methods (namely Kernel Logistic Regression) are used to infer interpretation rules. More precisely, two different probabilistic models are introduced: one to infer fish age from otolith images and a second one aiming at evaluating whether or not a given otolith growth pattern is realistic w.r.t. training examples. These probabilistic models provide us with the basis for coping with three different issues: the automated estimation of fish age from otolith images, the estimation of individual otolith growth patterns, and the definition of a confidence measure of otolith interpretations. These computer-assisted ageing tools are validated for a dataset of plaice otoliths.
\end{abstract}

Keywords: Statistical learning; Otolith interpretation; Otolith image analysis; Computer-assisted fish age and growth analysis 


\section{Problem statement and related work}

The acquisition of fish age and growth data from the analysis of calcified structures is at the core of stock assessment and marine Ecology issues. Fish otoliths are among the most commonly used structures for age determination. As illustrated in Fig.1 for a plaice otolith, otoliths are formed through an accretionary process, which may result in an alternation of translucent and opaque rings. For many species, seasonal rings can be observed. Hence, fish age can be estimated by counting these rings, while the estimation of otolith growth resorts to measuring the distance between the otolith center and the successive seasonal growth rings. One should stress that the analysis of otolith microstructures may also be considered to analyze daily growth increments. In this paper, the emphasis is given on seasonal growth rings, though the presented methodology is generic and cold be applied to the analysis of otolith microstructures.

[Figure 1 about here.]

Assessment or ecological issues typically require the interpretation of several thousand of calcified structures a year per stock or species. This tedious task is achieved by expert readers. Precision and accuracy issues are of key interest since age and growth data serves as the basis for further analysis: for instance, this data is used to estimate biological parameters (abundance, mortality,...) (Mesnil, 2003), and they also provide the time reference to calibrate other measures (e.g., chemical signatures) extracted from the calcified structures (Pontual and Geffen, 2003). Therefore, there is a need for defining protocols and tools targeted at quality assurance and quality control (Morison et al., 1998).

Computer-assisted tools provide means for the standardization of objective age reading techniques with a view to improving accuracy and precision levels. A va- 
riety of solutions can be designed to help in the acquisition of age and growth data (for instance, the automation of the acquisition of series of otolith images(Ogor and Fablet, 2004) or the storage and management of bases of images of interpreted calcified structures (CS) (Morison et al., 1998; Ogor and Fablet, 2004)) or to automate this task thansk to the estimation of fish age and growth estimation from CS images (Fablet et al., 2004b; Guillaud et al., 2002; Robertson and Morison, 1998; Troadec et al., 2000). The research effort has been mainly focused on the automation of the estimation of fish age and growth. Two broad categories of approaches can be distinguished: 1D methods (Lagardère and Troadec, 1997; Welleman and Storbeck, 1995) restrict their analysis to an intensity profile extracted along a given reading axis, while 2D techniques (Guillaud et al., 2002; Troadec et al., 2000) are aimed at providing a 2D segmentation of growth rings. While being far more robust, proposed 2D techniques are still limited to the detection of likely growth rings, and do not cover the interpretation task which requires a priori information to cope with false rings ro checks. The study by Robertson and Morrison (Robertson and Morison, 1998) was the first attempt to benefit from readers' experience to design a better system based on neural networks. Though promising, the reported results highlight the need for a better extraction of the information conveyed by otolith images. Though promising, the reported results highlight that the exploited image features (Fourier coefficients of the intensity signal along a given axis) do not to bring significant improvement for age estimation compared to other features (eg, otolith weight, fish size and sex). This somewhat contradicts the interpretation protocols followed by experts, who mainly rely on the interpretation of the internal otolith macrostructures to estimate the age. Hence, there is need for an improved automated extraction of the information conveyed by otolith images in terms of alternation of translucent and opaque rings. 
In this paper, a statistical learning strategy is also adopted and it is assumed that one can benefit from experts' experience by means of bases of interpreted otolith images. Whereas Robertson and Morrison only focus on fish age estimation from otolith images, two other applications are investigated: the estimation of individual growth patterns from otolith images and the definition of indicators of the reliability of readers' interpretation. To our knowledge, our work is the first attempt to provide such tools for computer-assisted aging. The key point of this work is to state the considered within a pattern classification framework, so that it benefits from the recent advances in statistical learning (Bishop, 1995; Scholkopf and Smola, 2002; Zhu and Hastie, 2001). They provide us with the methodological background to infer interpretation rules from training examples. This paper is organized as follows. Section 2 points out the key ideas of kernel-based statistical learning. Section 3 presents the information extraction stage within otolith images and the application to fish age estimation. The analysis and the estimation of individual otolith growth patterns is discused in Section 4. In Section 5, the design of a confidence measure of readers' interpretation is detailed. Results for plaice otolith samples are reported in Section 6 and Section 7 provides concluding remarks and an outline for future work.

\section{Statistical learning using kernel methods}

Age and growth analysis from otolith images is regarded as a pattern classification issue. More precisely, two main issues are considered: mapping otolith images to age groups and evaluating whether or not a given otolith growth pattern is realistic. In the first case, the classes are given by the age group and numerical descriptors will be extracted from otolith images to define a feature space. In the second case, 25 it will resort to a binary (realistic vs. non-realistic) classification issue, where the 
feature space will be defined from otolith growth patterns. Thus, in both cases, a generic classification issue is considered: assigning a class to observed feature vectors.

To solve for this issue, kernel approaches are among the most efficient techniques (Scholkopf and Smola, 2002). The kernel principle is illustrated in Fig.2. The use of a kernel function can be viewed as a means to map the original feature space to a higher dimensional space, within which the considered classification issue should be easier solved for. Given a feature space $\mathcal{X}$, kernel approaches rely on the introduction of the so-called kernel function $K$. Its key feature is to be defined as a dot product within a mapped space:

$$
\forall\left(x, x^{\prime}\right) \in \mathcal{X}^{2}, K\left(x, x^{\prime}\right)=<\Phi(x), \Phi\left(x^{\prime}\right)>
$$

where $\Phi$ defines a mapping from the initial feature space $\mathcal{X}$ to the transformed one $\Phi(\mathcal{X})$. A typical example of kernel function is given by the radial basis function: $K_{r b f}\left(x, x^{\prime}\right)=\exp \left(-\left(x-x^{\prime}\right)^{2} / 2 \sigma^{2}\right)$. It should be stressed that the mapping function $\Phi$ is never actually used. All the computations only involve its product. 15 This property is often referred to as the kernel trick.

[Figure 2 about here.]

In the following, the focus is given to two categories of kernel approaches: Support Vector Machines (SVM) and Kernel Logistic Regression (KLR).

\subsection{Support vector Machines}

SVMs have initially been developed by Vapnik (Vapnik, 1998) for character recog-

5 nition. Given a binary classification issue within the feature space $\mathcal{X}$ and a training set $\left\{\left(x_{i}, y_{i}\right) \in \mathcal{X} \times\{-1,1\}\right\}, \mathrm{SVM}$ techniques resort to the estimation of the hyperplane best separating as illustrated in 2. In the transformed feature space $\Phi(\mathcal{X})$, 
the hyperplane equation is given by: $\langle\Phi(w), \Phi(x)\rangle+b=0$, where $w$ is the vector normal to the hyperplane, and $b$ a translation parameter. Each half-space separated by this hyperplane refers to the two classes assigned according to the decision function $y=\operatorname{sign}(<\Phi(w), \Phi(x)>+b)$.

More formally, SVMs are maximum margin classifiers and resort to the following minimization (Scholkopf and Smola, 2002):

$$
(\hat{w}, \hat{b})=\arg \min _{(w, b)} \frac{1}{2}\|\Phi(w)\|^{2}+C \sum_{i} \eta_{i}
$$

under the constraints $\forall i, y_{i}\left(<w, x_{i}>+b\right) \geq 1-\eta_{i}$ and $\eta_{i} \geq 0$. Variables $\eta_{i}$ are measures of the errors of the classification of the data $\left(x_{i}, y_{i}\right)$ : Thus, the term $\sum_{i} \eta_{i}$ is a complexity-related penalty term, and the constant $C$ a regularization parameter used to balance the maximization of the margin against this complexity term.

Solving for this minimization issue comes to select support vectors $\left\{x_{j}^{S V}\right\}$ within the training data $\left\{x_{i}\right\}$ and to estimate their weights $\left\{\alpha_{j}\right\}$ such that the hyperplane vector $w$ is given by $\Phi(w)=\sum_{j} \alpha_{j} \Phi\left(x_{j}^{S V}\right)$. Hence, the binary decision function over $\mathcal{X}$ is defined as:

$$
d_{S V M}(x)=\operatorname{sign}\left(\sum_{j} \alpha_{j} K\left(x_{j}^{S V}, x\right)+b\right)
$$

5 The parameters of a SVM model are the supports vectors $\left\{x_{j}^{S V}\right\}$, the weights $\left\{\alpha_{j}\right\}$, the bias $b$ and the kernel function $K$.

\subsection{Kernel Logistic regression}

Contrarily to SVMs, kernel logistic regression (KLR) is aimed at estimating a probabilistic output $P_{K L R}(y(x)=1)$ of the classification function. Using a logistic model, $P_{K L R}(y(x)=1)$ is set as (Zhu and Hastie, 2001):

$$
P_{K L R}(y(x))=\left[1+\exp \left(\sum_{i} \alpha_{i} K\left(x_{i}, x\right)+b\right)\right]^{-1}
$$


where $\left\{x_{i}\right\}$ are the training data, $\left\{\alpha_{i}\right\}$ their estimated weights, and $b$ the estimated bias. One should point out that KLR does not share the important property of sparsity of SVMs: whereas in SVMs, only a few training samples are kept as the support vectors to actually define the classification function, all training samples contribute to the probabilistic output of the KLR model. However, the estimation of their parameters are issued from a slight adaptation of the SVM algorithms (Zhu and Hastie, 2001).

\subsection{Parameter estimation}

In practice, SVMs and KLR models require the selection of the appropriate kernel function $K$. In this paper the radial basis function is used: $K_{r b f}\left(x, x^{\prime}\right)=$ $\exp \left(-\left(x-x^{\prime}\right)^{2} / 2 \sigma^{2}\right)$ parametrized by the scale parameter $\sigma$. In addition, in both cases, it is required to estimate the optimal value of the regularization parameter $C$. Since no theoretical result exists to set $\sigma$ and $C$, one generally uses a crossvalidation strategy to determine the best parameter setting within a grid of predefined values of the regularization parameters $C$ and of the kernel parameter $\sigma$. The set of training samples $T$ is first split in a number $n$ (typically, $n$ is set to 5) of subsets $\left\{T_{1}, \ldots, T_{n}\right\}$ of equal number. The cross-validation procedure is then applied as follows. At iteration $k$ between 1 and $n$, a SVM (respectively KLR) model is estimated using the $\left\{T_{j}, j \neq k\right\}$ as the training samples. The performance of the estimated classifier is evaluated according to a loss function (for instance, the mean misclassification rate) for the samples of the subset $T_{k}$. The final stage consists in

5 computing the mean loss value for the different values of the regularization and kernel parameters, and in retaining the parameter setting with the best classification performance. 


\section{Automated fish age estimation}

In a first step, the aim is to infer fish age from otolith images. To state this issue

10 as a classification issue, it is first required to define an appropriate feature space $\mathcal{X}$. Therefore the computation a vector of numerical descriptors of the $2 \mathrm{D}$ image content is needed. Consequently, the extraction of relevant features from otolith images (in terms of alternation of translucent and opaque rings) is first detailed. Then, the application of a statistical learning scheme is described to automate fish 15 age estimation from the defined feature space.

\subsection{From 2D image content to $1 \mathrm{D}$ representation}

Since otolith interpretation is usually performed by experts along a predefined reading axis. For instance, for plaice otoliths, as shown by Fig.1, experts mainly consider the zone corresponding to the greatest growth. Consequently, our automated analysis the analysis of the content of otolith images can then be restricted to a region around the main reading axis. Let us stress that the proposed approach can be easily extended to several reading axes if required.

[Figure 3 about here.]

A template-based approach inspired from (Troadec et al., 2000) is then exploited to extract a one-dimensional signal depicting the 2D image content within an angular sector around the considered reading axis. More precisely, as illustrated in Fig. 3 given the otolith center 0 and an angular sector $\mathcal{S}$, the following 1D signal $s_{\mathcal{S}}$ is computed:

$$
s_{\mathcal{S}}(\alpha)=\operatorname{med}\left(I\left(O+\alpha * T_{\mathcal{S}}\right)\right)
$$

where $\operatorname{med}()$ is the median operator, $T_{\mathcal{S}}$ is the template model used within $\mathcal{S}, I$ the image intensity function, and $\alpha$ a scaling factor between 0 and 1. As in (Troadec 
et al., 2000), the template model $T_{\mathcal{S}}$ is given by the sampled external otolith shape within $\mathcal{S} . s_{\mathcal{S}}(\alpha)$ is then the median intensity value along the template $T_{\mathcal{S}}$ scaled by $\alpha$ w.r.t. the otolith center $O$. Since the scaling of the external otolith shape w.r.t. the otolith center provides a good approximation of the shape of the seasonal rings (Troadec et al., 2000), $s_{\mathcal{S}}$ depicts oscillations corresponding to the alternation of translucent and opaque rings within $\mathcal{S}$.

\subsection{Peak-based representation}

Expert interpretations rely on the detection of ridge and valley structures corresponding to translucent and opaque rings. In order to detect ridge and valley structures within $\mathcal{S}$, one needs to extract the meaningful extrema of the signal $s_{\mathcal{S}}$. This is achieved by determining the zero-crossings of derivative of $s_{\mathcal{S}}$. Since otolith growth is not linear but rather exponential, $s_{\mathcal{S}}$ is not stationary in terms of frequency content. It is then needed to adapt the scales at which the derivative of $s_{\mathcal{S}}$ is computed. This is done by applying a convolution to a Gaussian kernel whose variance is deduced from an a priori exponential otolith growth model. This is similar in spirit to the demodulation scheme applied in (Lagardère and Troadec, 1997).

Given the positions of the detected maxima (or equivalently the minima) of $s_{\mathcal{S}}$ $\left\{t_{1}, \ldots ., t_{n}\right\}$, a new normalized peak-based signal $s_{\mathcal{S}}^{P B}$ is defined by:

$$
s_{\mathcal{S}}^{P B}(t)=\min \left\{\rho\left(t-t_{k} / t_{n}\right)\right\}_{k \in\{1, \ldots, n\}}
$$

where $\rho$ is a distance kernel. The computation of $\left\{t_{1}, \ldots ., t_{n}\right\}$ is based on the detection of the zero crossings of the first derivative of $s_{\mathcal{S}}^{G}$, estimated by finite dif-

5 ferences. In practice, $\rho(u)=2 \operatorname{atan}(u) / \pi$. The resulting signal $s_{\mathcal{S}}^{P B}$ only depends on the extrema position as shown in Fig.4. In particular, compared to $s_{\mathcal{S}}^{G}, s_{\mathcal{S}}^{P B}$ is invariant to the variability of intensity ranges over images. 
[Figure 4 about here.]

\subsection{Application to fish age estimation}

10 tent of the otolith is rather high-dimensional (typically about 1000 dimensions). Hence, the straightforward application of SVM and KLR techniques is not feasible. A Principal Component Analysis (PCA) is therefore applied to retain only the first $N_{P C A}$ principal components (typically, $N_{P C A}<100$ ).

15 $n$ to an age group given its feature vector $x^{n}$. To cope with this multi-class issue, binary SVM and KLR models are extended to multi-class classification. A one vs. all strategy (Scholkopf and Smola, 2002) is used. It comes to train several binary classifiers, using a given age group as the first class and merging all the other age groups to define the second class. The outputs of these binary classifiers are combined according to a simple voting rule to lead to am ulti-class classification. When using KLR models, let us denote by $P_{A g e}(. \mid A=a)$ the resulting probabilistic model for the age class $a$.

The reader is referred to (Fablet et al., 2004b) for a more detailed description of the training issues, especially in terms of improvement of the generalization properties of the learned model.

\section{Analysis and estimation of individual growth patterns}

In addition to inferring fish age from otolith images, a key application is to esti5 mate individual otolith growth patterns and at evaluating the relevance of otolith interpretations w.r.t. previously interpreted samples. Both tasks require the development of an appropriate otolith growth model. 
Otolith growth is usually modeled using some a priori mean model, such as exponential and polynomial models, and the associated statistical distribution charof $\Delta g$ to define this feature vector is motivated by the key information brought by growth increments to discriminate actual growth patterns from erroneous ones. Let us stress that the length of the feature vector $X_{g}$ depends on the number of growth rings. Therefore, one model $P_{G r}(. \mid A=a)$ is learned for each age class $a$. acterizing the variability of the studied growth phenomena. This category of statistical models is not suited for the issues considered in this paper. It rather comes to evaluate whether or not a given growth pattern is likely to be an actual one. Due to the huge natural variability as illustrated in the experiments (see Section 6), the distance to a simple mean a priori model is not sufficient to tackle this binary classification issue. Therefore, a statistical learning scheme is exploited in order to train a system from labeled (realistic vs. non-realistic) examples of individual growth patterns.

\subsection{Statistical modeling of individual growth patterns}

Let us denote by $g$ an otolith growth pattern defined by the sequence of the distances $\left\{g_{1}, \ldots, g_{A}\right\}$ from the otolith center to the translucent (or equivalently opaque) rings. Our aim is to evaluate the likelihood $P_{G r}(g)=P(g$ is realistic $)$.

The probabilistic otolith growth model $P_{G r}(g)$ is estimated by statistical learning from a dataset of growth patterns labeled as realistic or non-realistic. Let us denote by $\mathcal{G}$ this dataset. Kernel logistic regression is applied using the feature vec25 tor $X_{g}=\{g, \Delta g\}$ with $\Delta g=\left\{g_{i}-g_{i-1}\right\}$ for any sample $g \in \mathcal{G}$. The computation

The relevance of the learned models $P_{G r}(. \mid A)$ depends on the ability of the samples in $\mathcal{G}$ to convey enough information to discriminate realistic growth patterns from erroneous ones. Given a set of otolith images interpreted by an expert, it 
is assumed that training samples for the "realistic" class are provided, but it is also needed to determine examples for the "non-realistic" class. The following procedure is exploited. For all the training images, the signal $s_{\mathcal{S}}(\alpha)$ as defined in Eq. 5 is extracted, and the positions of its local maxima $\left\{t_{i}\right\}_{i \in\left[1, N_{t}\right]}$ are computed. From this position set $\left\{t_{i}\right\}$, the $2^{N_{t}}$ subsets obtained by selecting from 1 to $N_{t}$ ring positions in $\left\{t_{i}\right\}$ are generated and the associated growth configurations are computed. From this set of $2^{N_{t}}$ growth configurations, all those different from the expert interpretation are kept as samples for the class "non-ralistic". This scheme is illustrated in Fig.5

[Figure 5 about here.]

For each age class $a$, the probabilistic model $P_{G r}(. \mid A=a)$ is estimated from the resulting training set using a cross-validation scheme as explained in Section 2.

\section{4.2 Automated estimation of individual growth patterns}

The definition of the probabilistic otolith growth models $\left\{P_{G r}(. \mid A=a)\right\}$ is first applied to the estimation of individual growth patterns from otolith images.

Given an otolith image $I$, the first step comes to extract the $s_{\mathcal{S}}(\alpha)$ as defined in Eq.5 and to compute the positions $\left\{t_{i}\right\}$ of its local maxima. The age estimator introduced in Section 3 infers an age class $a(I)$ from $s_{\mathcal{S}}$. Then, one generates the set $\mathcal{G}(a(I))$ of all the growth configurations corresponding to the age class $a(I)$ which can be generated from the set of the candidate ring positions $\left\{t_{i}\right\}$. Growth patterns within $\mathcal{G}(a(I))$ are sorted according to their likelihood w.r.t. the growth model $P_{G r}(. \mid A=a(I))$. The one with the greatest likelihood provides the estimated otolith growth pattern $\hat{g}$ :

$$
\hat{g}=\arg \max _{g \in \mathcal{G}(a(I))} P_{G r}(g \mid A=a(I))
$$


Let us point out that this scheme could also be used as an interactive tool for the acquisition of otolith growth data for previously interpreted samples: given an expert estimation of age, the positions of the growth rings can be automatically recovered.

\section{Confidence measure of otolith interpretations}

Beyond the automated estimation of fish age and growth from otolith images, the computation of a confidence measure is investigated to evaluate the reliability of otlith interpretations. As illustrated by Fig. 5 for two different interpretations of the same otolith, the aim is to define a measure pointing out that the top interpretation is unlikely, while the bottom one seems correct.

Formally, the definition of this confidence measure is viewed as the evaluation of the likelihood $P_{\text {Conf }}(g$ is relevant $\mid I)$ that a given interpretation (or growth pattern) $g$ is relevant given the observed otolith image $I$. Exploiting Bayes rule, $P_{\text {Conf }}(g$ is relevant $\mid I)$ is written as :

$$
\begin{array}{r}
P_{\text {Conf }}(g \text { is relevant } \mid I) \propto P(I \mid g \text { is relevant }) \\
\cdot \quad P(g \text { is realistic })
\end{array}
$$

$P(I \mid g$ is relevant $)$ is aimed at determining how likely the image $I$ is correctly explained by the interpretation $g$ in terms of age and growth. As a first step, this likelihood is computed from the statistical model $P_{\text {Age }}(a(g) \mid$.) introduced in Section 3, with $a(g)$ the age estimate associated with the growth pattern $g$. Therefore, the peak-based representation $s_{\mathcal{S}}^{P B}$ is computed within an angular sector specified by the expert as detailed in Section 3, and the likelihood $P(I \mid g$ is relevant $)$ is evaluated as:

$$
P(I \mid g \text { is relevant })=P_{\text {Age }}\left(s_{\mathcal{S}}^{P B} \mid a(g)\right) .
$$


Similarly, $P(I \mid g$ is not relevant $)$ is defined as $1-P_{\text {Age }}\left(s_{\mathcal{S}}^{P B} \mid a(g)\right)$.

$P(g$ is realistic $)$ conveys a priori information on growth patterns. It is evaluated from the probabilistic growth model $P_{G r}(g \mid A=a(g))$, as introduced in Section 4. Besides, $P(g$ is not realistic $)$ is given by $1-P_{G r}(g \mid A=a(g))$.

$P_{\text {Conf }}(g$ is relevant $\mid I)$ is then expressed as follows:

$$
\begin{aligned}
P_{\text {Conf }}(g \text { is relevant } \mid I) & =P_{\text {Age }}\left(s_{\mathcal{S}}^{P B} \mid a(g)\right) \\
& \cdot P_{G r}(g \mid a(g)) \cdot Z^{-1}
\end{aligned}
$$

with the normalization factor $Z$ given by:

$$
\begin{aligned}
Z & =P_{\text {Age }}\left(s_{\mathcal{S}}^{P B} \mid a(g)\right) \cdot P_{G r}(g \mid a(g)) \\
& +\left(1-P_{\text {Age }}\left(s_{\mathcal{S}}^{P B} \mid a(g)\right)\right)\left(1-P_{G r}(g \mid a(g))\right)
\end{aligned}
$$

Given an image $I$ and an interpretation $g$, the closest $P_{\text {Conf }}(g$ is relevant) to one, the more relevant the interpretation. Conversely, the closest $P_{\text {Conf }}(g$ is relevant $)$ to zero, the less relevant the interpretation. Let us point out that this relevance measure needs to be interpreted with respect to the variability encountered within the training sets exploited to learn the probabilistic models $P_{A g e}$ and $P_{G r}$.

\section{Results}

\subsection{Otolith database}

[Figure 6 about here.]

[Figure 7 about here.]

Experimental results are reported for a database of 320 images of plaice otoliths from age group 1 to 6 caught during the fourth quarter in 1994 and 2000. The 
ground truth for fish age estimation is given by the interpretation of an expert. Due to the lower number of samples in age groups 5 and 6, these two groups are

In Fig. 8 is reported the classification results for the images of the test set (i.e., one third of the considered image dataset for each age group), the mean rate of correct classification (w.r.t. the expert ground truth) with SVMs and KLR is of $88 \%$. Not surprisingly, classification errors are more numerous for the older age 5 error is below or equal to one. This mean rate of correct age estimation is close to inter-expert agreement rates. This highlights the interest of the proposed scheme 
and the need for a carefully designed pattern recognition scheme to achieve good classification performance.

These results can be favorably compared to those obtained by previous approaches (Guillaud et al., 2002). In addition, the application to routine aging seems promising since these results are in the range of the levels of agreement observed between expert readers. The reader is referred to (Fablet et al., 2004b) for a more detailed evaluation of the proposed approach for automated ageing and a comparison between different feature types (frequency vs peak-based features) and statistical classifiers (neural nets vs. SVMs). This evaluation stresses that kernel-based learning outperforms neural networks, and that the proposed feature set leads to significant improvements (greater than $10 \%$ in terms of mean correct classification rate) compared to previous work.

\subsection{Estimation of individual growth patterns}

[Figure 9 about here.]

As an illustration of the proposed approach for the estimation of individual growth patterns, Fig.9 depicts an otolith image belonging to the age class 4 . It reports the candidate ring positions which are provided by the maxima of the template-signal $s_{\mathcal{S}}$, the expert interpretation. From the set of growth configurations of age class 4 , the configuration with the greatest likelihood value $P_{G r}(g \mid a=4)$

5 is depicted. As shown by Fig.9, the actual growth pattern is successfully estimated. Similarly to the age estimation application, the probabilistic growth model is trained from the two third of the considered plaice datasets. 
A quantitative evaluation of the proposed approach has also been performed. Given the test set of otolith images, the statistical learning approach has been applied to the estimation of the growth pattern, given the age estimate provided by the expert. For $95 \%$ of the samples, one retrieves the same growth interpretation as the one provided by the expert.

\subsection{Confidence measure of otolith interpretations}

[Figure 10 about here.]

In Fig.10 several examples of the computation of a confidence measure of the interpretation of otolith growth rings for plaice otoliths are displayed. For each image, the confidence measure is compputed for two different interpretations the expert one and an erroneous one. As expected, the confidence measure is greater than 0.5 in the first case and closer to zero in the latter. Besides, Fig.10 shows that the computation of these confidence measures may assist the reader in ambiguous cases. For instance, for the second and third examples reported in Fig.10, it helps in interpreting the first growth ring. This case illustrates the potential interest of this confidence measure for routine ageing, where the interpretation of the first ring is known to be one of the key issue.

These different examples stress that the computation of these confidence measures of otolith interpretation could be used for routine ageing issues: either as an 5 interactive tool to assist the expert for ambiguous cases, or as an automated verification tool to detect erroneous or unlikely interpretations within a set of interpreted otolith sets with a view to asking for a second reading. 


\section{Conclusion}

This paper has discussed the use of statistical learning techniques, namely kernel approaches, to benefit from a training set of interpreted data in order to cope with computer-assisted fish age and growth analysis. Information extraction within otolith images has been regarded as the computation of a template-based 1D signal summing up the image content within a predefined area of interest (typically defined as an angular sector around the main reading axis). Using statistical learning, this signal can be used to infer fish age. New probabilistic models of otolith growth, learned from otolith samples interpreted by an expert, have been introduced to discriminate realistic growth patterns from erroneous one. These models are exploited to estimate individual otolith growth patterns and to compute confidence measures of otolith interpretations.

The evaluation of the proposed approach on a set of several interpreted plaice otoliths from the fourth quarter validates the proposed models and demonstrates their key interest for routine age and growth estimation, especially in the context of quality assurance and quality control in age determinations. There are a number of potential applications such as the complete automation of the acquisition of age and growth data for otolith subsets, the automation of the creation of bases of CS images and of the associated interpretations from aged CS datasets, or computeraided CS interpretation. At the operational level (for instance, for the determination of age structures used for stock assessment), the key benefits expected from these tools are to reduce the cost of the acquisition of age data from CS, while improving data quality (mainly, in terms of precision) and data traceability (both in terms of 5 age determinations and in terms of growth ring interpretations through the automation of the creation of databases of images of interpreted materials).

Some potential limits of the application of statistical learning strategies should 
also be pointed out. Since they rely on the sets of interpreted CS structures with a view to automatically inferring the associated interpretation rules and to applying these rules to new samples, the capability of the trained systems to efficiently extrapolate from the training set obviously depends on the features and the associated spread within the training set w.r.t. to those within the test set. For instance, if significant changes occur in otolith growth for a given stock, new training sets might be needed to apply these techniques from one year to another. This is of 15 key importance and should be evaluated in future work. Similarly, such techniques are not able to cope with age classes not present within the training sets and their performance in terms of precision and accuracy rely on the quality of the training data. In most cases, the latter provides upper bounds for the performances of the trained system. One might point out that it should be preferred to build training sets from known age materials, if available. Consequently, the transfer to the operational level of the proposed methodology should be evaluated on a species- and stock-specific basis. While it should first provide a relevant alternative and support to age determinations for rather "easy-to-read" species such as flat fish otoliths, more complex cases need to be investigated. For instance, similar types of probabilistic otolith growth models should be considered for situations where the growth analysis needs to be performed along polylines or curved axis. Otherwise, whereas the proposed peak-based representation of the content of otolith images and the exploited learning strategies can be regarded as generic, the emphasis should be given on the information extraction stage (for instance, for the detection of the 5 growth rings) to cope with samples like cod or hale otoliths. Besides, the issue of combining the proposed approaches to schemes dedicated to 2D ring segmentation (Guillaud et al., 2002; Troadec et al., 2000) is also of particular interest. 


\section{Acknowledgements:}

The author wishes to thank A. Benzinou for fruitful discussion on the templatebased model and N. Le Josse for his input to this work. Besides, the author is thankful to A. Ogor for his help in the acquisition of the image dataset.

\section{References}

Bishop, C. (1995). Neural Networks for Pattern Recognition. Oxford University Press.

Fablet, R., Josse, N. L., and Benzinou, A. (2004b). Automatic fish ageing from otolith images using statistical learning. In Proc. of 17th Int. Conf. on Pattern Recognition, ICPR'04, volume 4, pages 503-506, Cambridge, UK.

Guillaud, A., Benzinou, A., Troadec, H., Rodin, V., and Bihan, J. L. (2002). Autonomous agents for edge detection and continuity perception on otolith images. Image and Vision Computing, 20(13-14):955-968.

Lagardère, F. and Troadec, H. (1997). Age estimation in common sole solea solea larvae: validation of daily increments and evaluation of a pattern recognition technique. Marine Ecology Progress Series, 155:223-237.

Mesnil, B. (2003). Manual of Fish Sclerochronology, chapter Some uses of individual age data. Eds J. Panfili, H. de Pontual, H. Troadec and P.J. Wright, Editions Ifremer.

Morison, A., Robertson, S., and Smith, D. (1998). An integrated system for production fish aging: image analysis and quality assurance. North American Journal of Fisheries Management, 18:587-598.

Ogor, A. and Fablet, R. (2004). Tnpc software: digital processing of calcified structures. www.ifremer.fr/lasaa.

5 Pontual, H. de and Geffen, A. (2003). Manual of Fish Sclerochronology, chapter Otolith microchemistry. Eds J. Panfili, H. de Pontual, H. Troadec and P.J. Wright, Editions Ifremer. 
Robertson, S. and Morison, A. (1998). Development of an artificial neural network for automated age estimation. Technical Report 98/105, Marine and Freshwater Resources Institute.

Scholkopf, B. and Smola, A. J. (2002). Learning With Kernels: Support Vector Machines, Regularization, Optimization and Beyond. MIT Press.

Troadec, H., Benzinou, A., Rodin, V., and Bihan, J. L. (2000). Use of deformable templates for otolith 2D growth ring detection by digital image processing. Journal of Fisheries Research, 46(1-3):155-163.

Vapnik, V., editor (1998). Statistical learning theory. Wiley.

Welleman, H. and Storbeck, F. (1995). Automatic ageing of Plaice otoliths by means of image analysis. In Secor, D., Dean, J., and Campana, S., editors, Recent developments in Fish Otolith Research, pages 271-282. Univ. of South Carolina Press.

Zhu, J. and Hastie, T. (2001). Kernel logistic regression and the import vector machine. In Int. Conf. on Neural Information Processing Systems, Vancouver. 


\section{List of Figures}

1 Illustration of plaice otolith interpretation for a 4 year old individual. The otolith image has been acquired using a binocular microscope under transmitted light. The interpretation of the winter translucent rings is displayed by the markers set on the radial drawn on the main reading axis. . . . . . . . . . . . . . . . . . . .

2 Key principle of kernel-based classification: map the original feature to a higher-dimensional feature space via the kernel function $\Phi$ and determine the separating hyperplane with maximum margin (from (Scholkopf and Smola, 2002)). . . . . . . . . . . . . . .

3 Template-based approach aimed at extracting a 1D representation of the image content within a region of interest. . . . . . . . . . . .

$4 \quad$ Illustration of the peak-based representation for the otolith image depicted in 1: a) signal $s_{\mathcal{S}}$ and the extracted maxima positions $(\mathbf{\nabla}$ markers), b) associated peak-based representation $s_{\mathcal{S}}^{P B} \ldots \ldots .$.

5 Creation of the training set for probabilistic otolith growth modeling: a) otolith image with the positions $\left\{t_{i}\right\}$ (red $\downarrow$ ) of the maxima of the signal $s_{\mathcal{S}}(\alpha)$ (cf. Eq.5), the ring position for the actual otolith interpretation (blue $\bullet$ ) and the ring positions for the nonrealistic one (magenta $\mathbf{\square}$ ), b) growth patterns examples for the two classes standing for realistic and non-realistic growth patterns. . .

6 Plaice otolith dataset: a) age distribution of the database of 320 plaice otoliths, $b$ ) illustration of growth variability within the considered dataset. The otolith growth patterns (distance from the otolith center to the center as a function of the age) are displayed for the 320 plaice otoliths. . . . . . . . . . . . . . . . . .

7 Sample images within the processed dataset of plaice otolith images. 30

Plot of the ageing errors from age group 1 to age group $5+$ for the plaice test set. . . . . . . . . . . . .

9 Illustration of the estimation of the growth pattern for a plaice otolith within age class $a(I)=4:$ a) the image I the expert interpretation (red $\circ$ ) is depicted the extracted set of candidate growth ring positions (cyan $\diamond)$ and the estimated ring positions (magenta -) (see Section 4 for further details), b) plot of the growth patterns for the expert interpretation and the estimated growth pattern. . . 
10 Computation of confidence measures for otolith interpretations: the first column reports the images of plaice otoliths with two different interpretations (the expert one and an erroneous one), while the second raw plots the associated growth pattern and the associated confidence measure $p=P_{\text {Conf }}(g$ is relevant $\mid I)(c f$. Eq.10). . . . . . . . . . . . . 


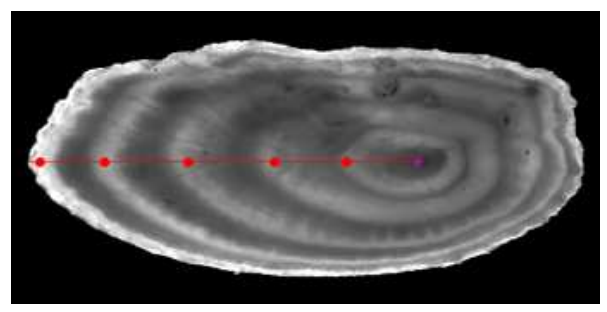

Figure 1: Illustration of plaice otolith interpretation for a 4 year old individual. The otolith image has been acquired using a binocular microscope under transmitted light. The interpretation of the winter translucent rings is displayed by the markers set on the radial drawn on the main reading axis. 


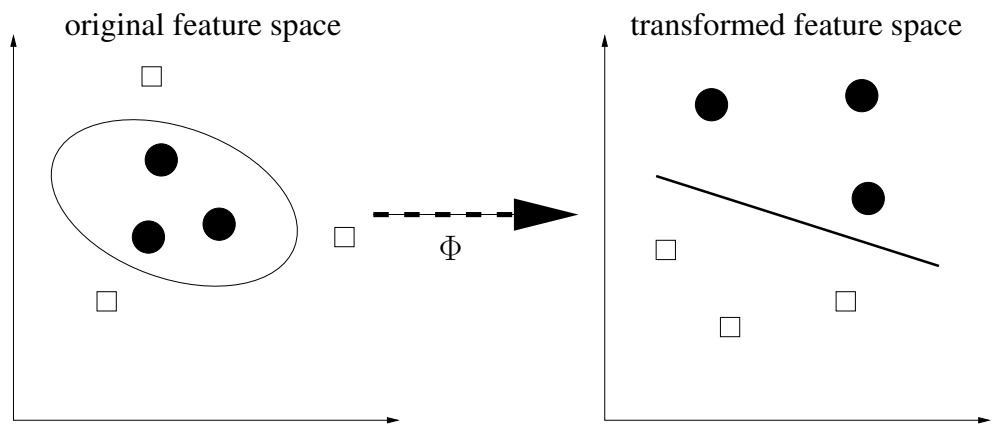

Figure 2: Key principle of kernel-based classification: map the original feature to a higher-dimensional feature space via the kernel function $\Phi$ and determine the separating hyperplane with maximum margin (from (Scholkopf and Smola, 2002)). 


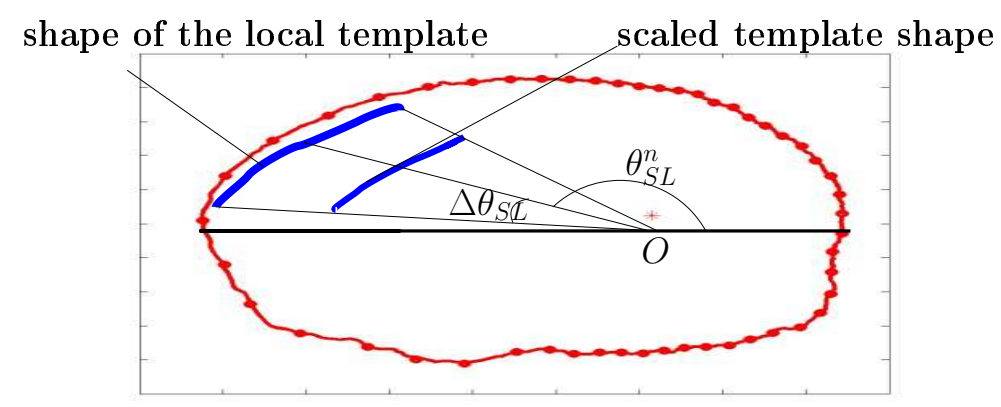

Figure 3: Template-based approach aimed at extracting a 1D representation of the image content within a region of interest. 
a) peak positions

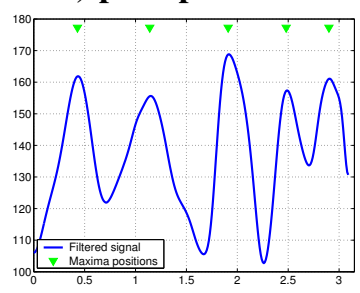

b) peak-based representation

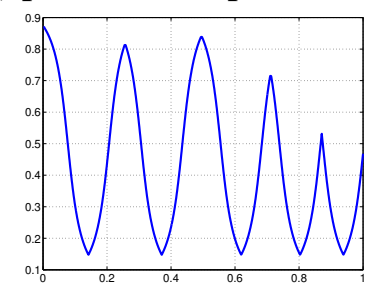

Figure 4: Illustration of the peak-based representation for the otolith image depicted in 1: a) signal $s_{\mathcal{S}}$ and the extracted maxima positions ( $\boldsymbol{\nabla}$ markers), $b$ ) associated peak-based representation $s_{\mathcal{S}}^{P B}$. 
a) otolith image

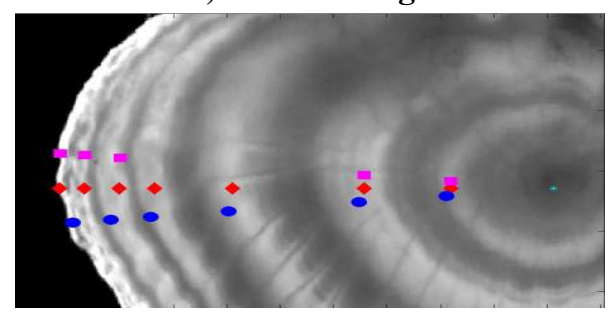

b) growth patterns

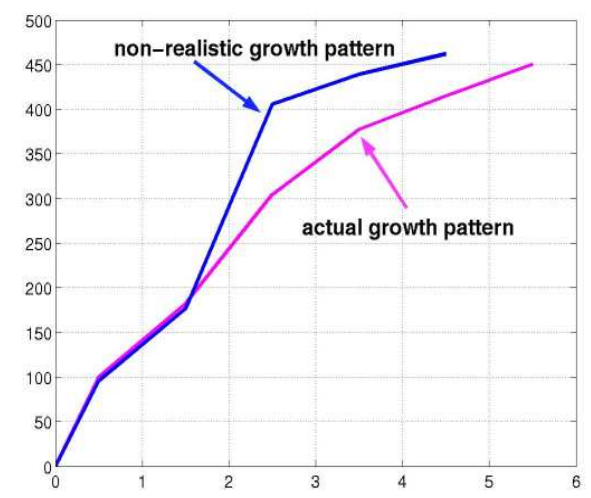

Figure 5: Creation of the training set for probabilistic otolith growth modeling: a) otolith image with the positions $\left\{t_{i}\right\}$ (red ) of the maxima of the signal $s_{\mathcal{S}}(\alpha)$ (cf. Eq.5), the ring position for the actual otolith interpretation (blue $\bullet$ ) and the ring positions for the non-realistic one (magenta $\mathbf{0}$ ), b) growth patterns examples for the two classes standing for realistic and non-realistic growth patterns. 
a) age composition

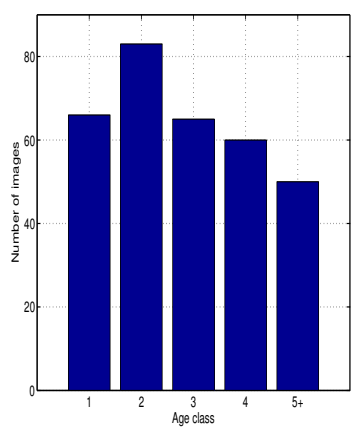

b) growth variability

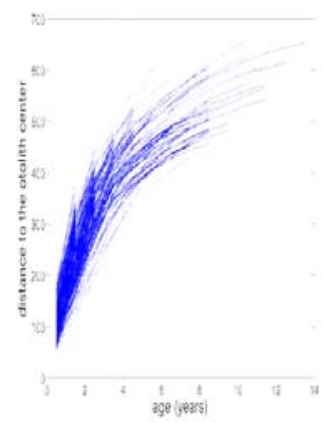

Figure 6: Plaice otolith dataset: a) age distribution of the database of 320 plaice otoliths, b) illustration of growth variability within the considered dataset. The otolith growth patterns (distance from the otolith center to the center as a function of the age) are displayed for the 320 plaice otoliths. 

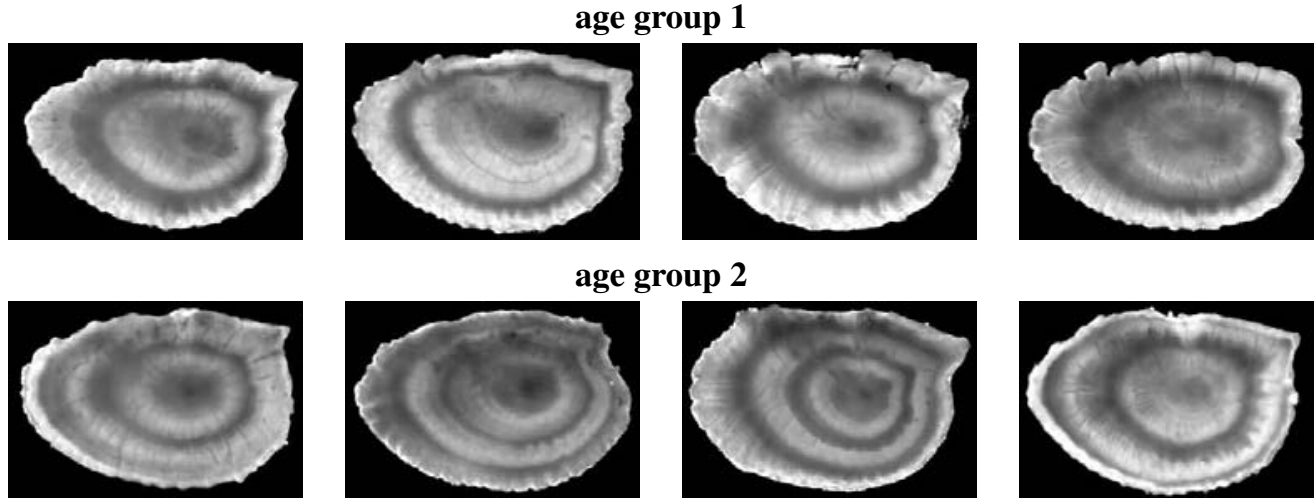

age group 3
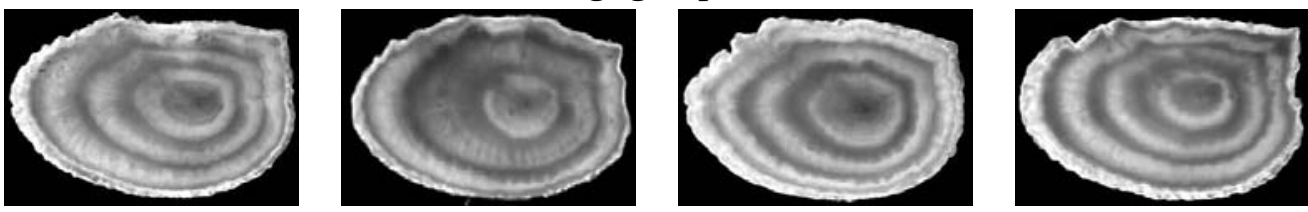

age group 4
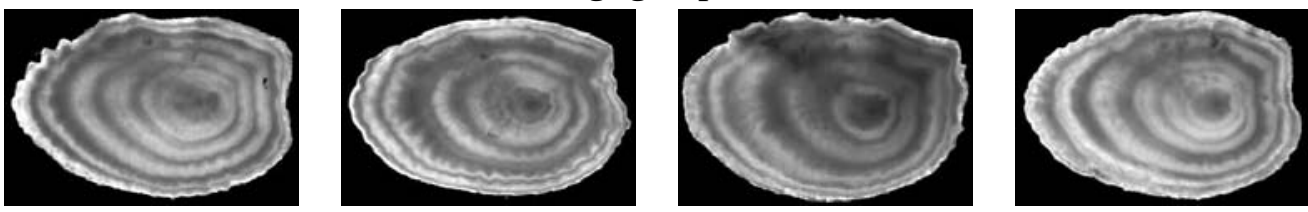

age group 5+
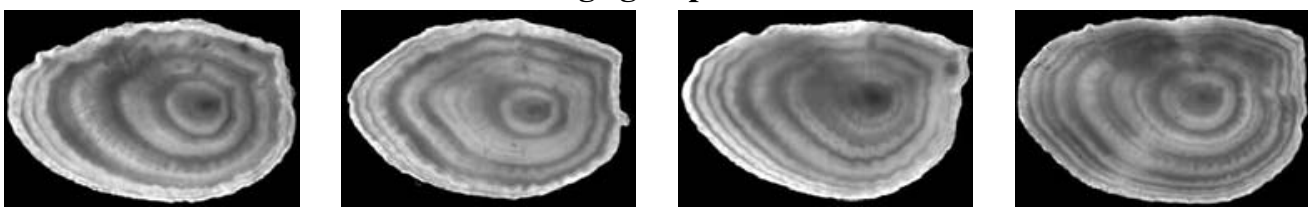

Figure 7: Sample images within the processed dataset of plaice otolith images. 


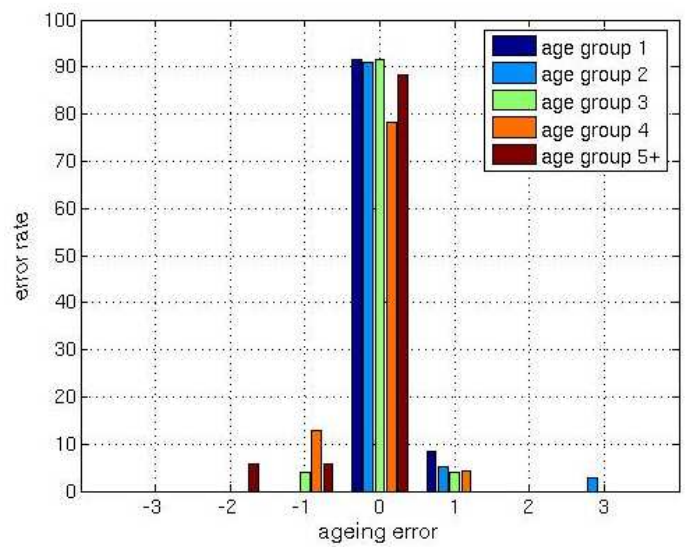

Figure 8: Plot of the ageing errors from age group 1 to age group 5+ for the plaice test set. 
a) otolith image

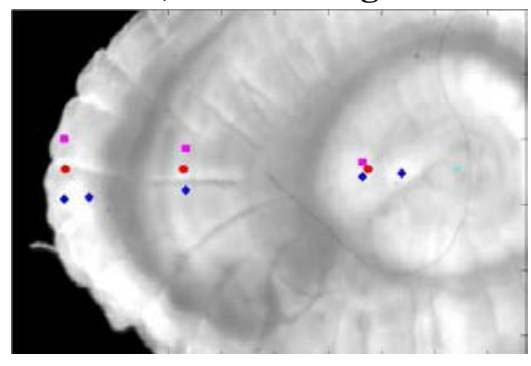

a) growth patterns

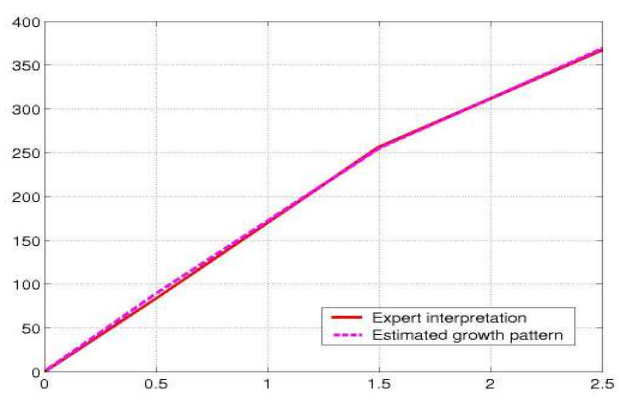

Figure 9: Illustration of the estimation of the growth pattern for a plaice otolith within age class $a(I)=4:$ a) the image I the expert interpretation (red $\circ$ ) is depicted the extracted set of candidate growth ring positions (cyan $\diamond)$ and the estimated ring positions (magenta $\mathbf{\square}$ ) (see Section 4 for further details), b) plot of the growth patterns for the expert interpretation and the estimated growth pattern. 

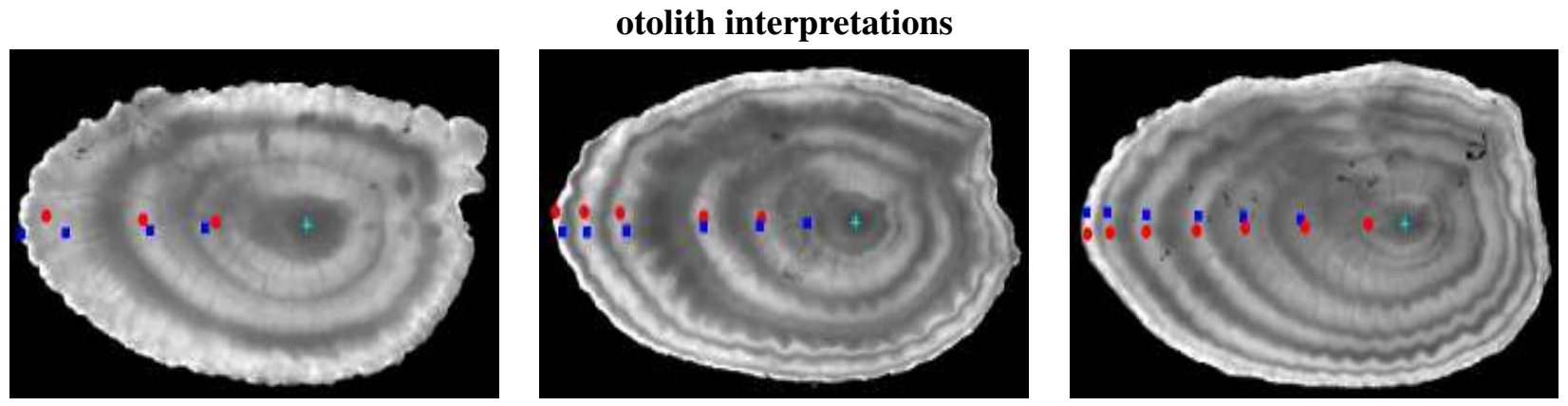

growth patterns and associated confidence measures
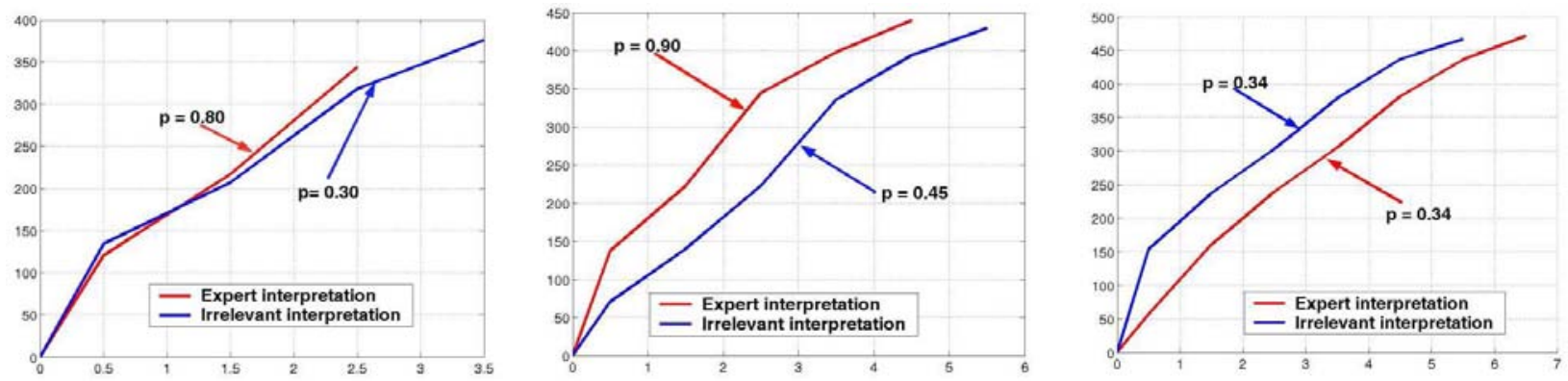

Figure 10: Computation of confidence measures for otolith interpretations: the first column reports the images of plaice otoliths with two different interpretations (the expert one and an erroneous one), while the second raw plots the associated growth pattern and the associated confidence measure $p=P_{\text {Conf }}(g$ is relevant $I)$ (cf. Eq.10). 